doi: http://dx.doi.org/10.5892/ruvrv.2012.101.218233

\title{
Novos conceitos sobre a fisiologia da hemostasia
}

\author{
Evandra Straza RODRIGUES ${ }^{\mathrm{I}}$ \\ Andrielle CASTILHO-FERNANDES ${ }^{\text {II }}$ \\ Dimas Tadeu COVAS ${ }^{\text {III }}$ \\ Aparecida Maria FONTES ${ }^{\text {IV }}$
}

I Doutoranda pelo Departamento de Biociências, Faculdade de Ciências Farmacêuticas de Ribeirão Preto (FCFRP) -
Universidade de São Paulo (USP), Instituto Nacional de Ciência e Tecnologia em Células-Tronco e Terapia Celular
(INCTC) - Brasil, evandra@ @emocentro.fmrp.usp.br
II Doutora pelo Departamento de Clínica Médica - Modalidade Biomédica, Faculdade de Medicina de Ribeirão Preto
(FMRP) - USP, INCTC - Brasil, andrielle_dcf@yahoo.com
III Professor Titular da Faculdade de Medicina de Ribeirão Preto (FMRP) - USP, INCTC - Brasil, dimas@fmrp.usp.br
IV Professora Doutora vinculada ao Departamento de Clínica Médica e de Genética - FMRP - USP, INCTC - Brasil,
fontesam@ @emocentro.fmrp.usp.br

RESUMO: A hemostasia envolve reações que impedem a perda de sangue, restauram a integridade vascular, e mantém condições fisiológicas que preservam a vida. O entendimento do sistema hemostático requer o conhecimento de um mecanismo complexo, controlado pelo endotélio vascular, micropartículas derivadas de diversos tipos de células, plaquetas, vias da coagulação sanguínea e fibrinólise. Recentes avanços no conceito de cascata da coagulação gerados por observações clínicas e experimentais demonstraram que o modelo da cascata clássica proposto na década de 60 não reflete completamente a hemostasia in vivo. Dessa forma, este artigo aborda os diversos componentes do sistema hemostático e fibrinolítico, o endotélio, as micropartículas e as plaquetas. Em seguida aborda a cascata clássica e as mudanças que ocorreram no entendimento do processo fisiológico da coagulação que conduziram ao modelo atual da cascata da coagulação baseado nas superfícies celulares.

Palavras-chave: Cascata da coagulação, superfícies celulares, hemostasia, fibrinólise.

\section{New concepts on the physiology of hemostasis}

\begin{abstract}
Haemostasis comprises a series of reactions that preventing blood loss, restore vascular integrity and maintains physiological conditions that preserve life. Understanding of hemostatic system includes the acknowledgement of complex mechanism controlled by the vascular endothelium, platelets, the coagulation pathway and fibrinolysis. Recent advancements in the comprehension of concept of the coagulation cascade, by clinical and experimental observations demonstrated that the classical cascade model doesn't completely reflect the process of hemostasis in vivo. Then, this article discusses several hemostatic and fibrinolic components, the endothelium, cellderived microparticles and platelets. Next, we described the classical cascade model and the changes that occurred in the understanding of physiologic processes of coagulation in order to be proposed the actual model of coagulation cascade based on the cell surfaces.
\end{abstract}

Key-words: Cascade of coagulation, coagulation, haemostasis, fibrinolysis.

\section{1- INTRODUÇÃO}

\author{
Hemostasia é o processo fisiológico \\ cujo principal objetivo é a manutenção da \\ integridade vascular e da fluidez do sangue
}

após uma lesão vascular permitindo o equilíbrio do sistema circulatório. Esse processo compreende interações complexas entre os vasos sanguíneos, plaquetas, proteínas da coagulação e $\quad$ o $\quad$ sistema 
fibrinolítico, os quais levam à formação do coágulo sanguíneo e posterior dissolução do mesmo após o reparo da lesão vascular ${ }^{68,45}$.

A princípio a interação desses componentes levou à segmentação da hemostasia em três fases distintas: 1) Primária; 2) Secundária ou Coagulação e 3) Terciária ou Fibrinólise ${ }^{39,}$ 17, 13. Contudo, vários avanços ocorreram no conhecimento dos mecanismos da coagulação sanguínea, o que mostrou a interdependência das fases hemostáticas. Diante deste contexto, essa revisão tem por objetivo abordar a evolução dos principais conceitos relacionados ao processo hemostático in vivo. Primeiramente abordamos os componentes do sistema hemostático: endotélio vascular, micropartículas e plaquetas. Em seguida, a hemostasia primária, a hemostasia secundária e a hemostasia terciária. $\mathrm{Na}$ hemostasia secundária, primeiro é descrito o modelo clássico da cascata que permite identificar as interações bioquímicas que ocorrem entre os diferentes fatores da coagulação sanguínea in vitro, suportando avaliações laboratoriais. Posteriormente, abordamos o novo modelo hemostático que é uma melhor representação da fisiologia da coagulação per se, baseado nas interações dos fatores de coagulação com as superfícies celulares.

\section{2- COMPONENTES DO SISTEMA HEMOSTÁTICO}

\section{1- Endotélio vascular}

O endotélio vascular é um tecido localizado entre o lúmen vascular e os constituintes da parede do vaso, capaz de liberar várias substâncias envolvidas no tônus vascular local e na coagulação sanguínea ${ }^{6}$.

Em seu estado normal, a superfície endotelial exerce uma função anticoagulante, favorecendo a dilatação vascular e contribuindo para manutenção da fluidez do sangue. Neste estado, as células endoteliais sintetizam moléculas que desempenham o papel de regular o fluxo sanguíneo e inibir a ativação e agregação das plaquetas. Entre elas podemos citar: a) glicosaminaglicanas (Heparan sulfato $)^{15}$; b) prostaciclina $\left(\mathrm{PGI}_{2}\right) ; \mathrm{c}$ ) prostaglandina; d) oxido nítrico (NO), que atua de forma sinérgica com a $\mathrm{PGI}_{2}$ e e) ectonucleotidases (ecto-ADPase) 49, 3, 30, 27 (Figura 1A).

Mediante uma lesão vascular, o endotélio sofre uma reprogramação bioquímica que induz à produção de um potente vasoconstritor (endotelina 1) e à formação de uma superfície prócoagulante. Neste caso, as células endoteliais secretam o fator tecidual (FT) e o fator de von Willebrand $(\mathrm{FvW})$ que atuam no processo da coagulação sanguínea $^{12,54}$ (Figura 1B).

O endotélio evita ainda a ativação excessiva da cascata da coagulação e a oclusão vascular, por meio da síntese de 
diferentes proteínas: a) proteína inibidora do fator tecidual (Tissue factor pathway inhibitor

- TFPI), que se associa ao fator Xa e inibe o complexo FT/VIIa/ $\mathrm{Xa}^{52}$; b) proteína $\mathrm{C}$, a qual junto com seu cofator (proteína $\mathrm{S}$ ) inativa os fatores Va e VIIIa; c) heparan sulfato, conhecido como glicosaminaglicana, que atua como cofator da antitrombina III (ATIII) e previne a ativação plaquetária e a coagulação intravascular; d) anexina $\mathrm{V}$, que impede o ancoramento dos fatores da coagulação

\section{Anticoagulante}

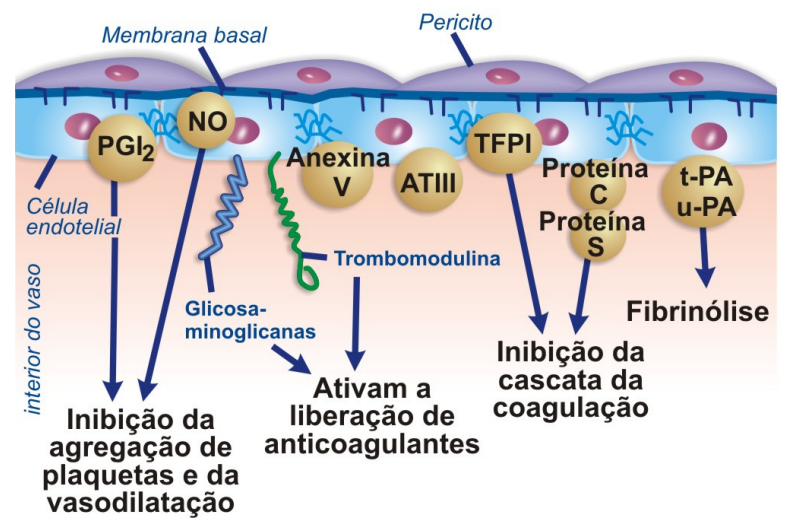

durante a sua ativação; e d) trombomodulina (TM) que inativa a trombina quando forma o complexo trombina-TM, que por sua vez impede a ativação das plaquetas e dos fatores V, VIII e fibrinogênio ${ }^{14}$. Além disso, a superfície do endotélio contém dermatan sulfato que se liga ao cofator II da heparina e promove a inibição da trombina ${ }^{12,49,22,78,54}$.

Figura 1 - Mediadores endoteliais da hemostasia. (A) Principais constituintes da função anticoagulante da célula endotelial: NO, PGI2, ATIII, TFPI, Proteína C, Anexina V, ativador tecidual do plasminogênio (tPA) e ativador do plasminogênio tipo-uroquinase (uPA). (B) Principais constituintes da função prócoagulante da célula endotelial: FvW, trombospondina, FT e inibidor do ativador do plasminogênio tipo 1 (PAI-1). Retirado de Hutton AR et al., 199975.

\section{2 - Plaquetas}

As plaquetas constituem fragmentos citoplasmáticos de 2,0 a 3,0 $\mu \mathrm{m}$ de diâmetro derivados do megacariócito da medula óssea e no vaso sanguíneo intacto se encontram em estado de repouso. Normalmente, as plaquetas apresentam uma frequência de 130.000 a 400.000 unidades $/ \mathrm{mL}^{62,} \quad 23$ e circulam no sangue periférico de 9-10 dias ${ }^{62}$.

Mediante a injúria vascular, as plaquetas são ativadas e se aderem ao endotélio, via ligação das glicoproteínas da superfície das plaquetas ao FvW produzido pelas células endoteliais ${ }^{62,23,73}$.

As plaquetas ativadas agem em diferentes etapas da hemostasia por meio da secreção de constituintes presentes em seus grânulos $\alpha$ e eletrodensos, entre eles: 1) proteínas com atividade de adesão e agregação plaquetária (FvW, fibrinogênio, fibronectina e trombospondina); 2) proteínas coagulantes (fatores V e XI); 3) inibidores de 
fibrinólise (PAI-1 e inibidor da plasmina); 4) neutralizante de heparina (fator plaquetário 4), 5) indutores da mitose (fator de crescimento derivado de plaquetas - PDGF), 6) inibidor da secreção de prostaciclina pelas células endoteliais ( $\beta$-tromboglobulina) e 7 ) indutores da vasoconstrição (serotonina e tromboxano A2 (TXA2) $)^{72,2,44,38 .}$

\section{3 - Micropartículas}

São pequenos fragmentos $(0,1-1,0$ $\mu \mathrm{m}$ de diâmetro) da membrana plasmática de plaquetas, leucócitos e células endoteliais. Estão presentes na circulação sanguínea, sendo as mais abundantes as derivadas de plaquetas, as quais desempenham importante papel na hemostasia ${ }^{34,25,11}$.

Em situações normais, as micropartículas produzem quantidades ínfimas de trombina, que ativam a proteína $\mathrm{C}$ e desempenha um papel anticoagulante, o que contribui para a manutenção do fluxo sanguíneo nos vasos ${ }^{5,76}$. Frente a uma lesão vascular, as micropartículas possuem efeito coagulante, já que secretam quantidades pouco maiores de trombina, que por sua vez ativam plaquetas e o fator VIII (FVIII). As micropartículas podem ainda induzir a ativação das plaquetas via transferência do TXA2, e de seu precursor, ácido araquidônico às plaquetas não ativadas ${ }^{77}$. Além disso, as micropartículas expressam receptores de alta afinidade para os fatores da coagulação VIII e
Va, contribuindo assim na formação do complexo protombinase ${ }^{26}$.

\section{4 - Fatores plasmáticos pró-coagulantes}

Os fatores plasmáticos prócoagulantes constituem a segunda linha de defesa do organismo após uma lesão vascular e exercem seu papel na hemostasia secundária ou coagulação sanguínea. Esses fatores descobertos entre 1940-1950 apresentam uma nomenclatura designada pelo Comitê Internacional de Trombose e Hemostasia que consiste no uso de números romanos de acordo com sua ordem de descoberta, seguido ou não pela letra "a", a qual indica a molécula em seu estado ativado $^{74}$. Os fatores plasmáticos perfazem 14 diferentes proteínas e esta regra é adotada para 11 deles, sendo o $\mathrm{Ca}^{2+}$ dificilmente denominado fator IV, assim como, para a precalicreína (PC) e o cininogênio de alto peso molecular (CAPM) esta nomenclatura não foi adotada.

Esses fatores apresentam também uma nomenclatura tradicional que pode estar relacionada com: a) proteína precursora; b) doença causadora da deficiência; c) pesquisador responsável pela descoberta ou ainda d) nome do paciente portador do fator deficiente. $\mathrm{O}$ peso molecular desses fatores pode variar de 40 a $340 \mathrm{kDa}$ e sua concentração no plasma pode variar de 0,2 a
$\mu \mathrm{g} / \mathrm{mL}$
(Tabela 
Tabela 1. Características dos Fatores da Coagulação Humana

\begin{tabular}{|c|c|c|c|c|}
\hline Nome Tradicional & $\begin{array}{l}\text { Nomenclatura } \\
\text { Preferida }\end{array}$ & $\begin{array}{l}\text { Peso Molecular } \\
\text { (Da) }\end{array}$ & $\begin{array}{l}\text { Concentração } \\
\text { Plasmática } \\
(\mu \mathrm{g} / \mathrm{mL})\end{array}$ & Função \\
\hline Fibrinogênio & Fator I & 340.000 & $2.600,0$ & Precursor \\
\hline Protrombina & Fator II & 72.000 & 100,0 & Zimogênio \\
\hline Fator tecidual & Fator III & 44.000 & NA & Cofator \\
\hline Cálcio & Fator IV & 40 & 100,0 & Cofator \\
\hline Proacelerina & Fator V & 330.000 & 6,6 & Procofator \\
\hline Proconvertina & Fator VII & 50.000 & 0,5 & Zimogênio \\
\hline Fator Anti-hemofílico & Fator VIII & 285.000 & 0,2 & Procofator \\
\hline Fator de Chistmas & Fator IX & 57.000 & 5,1 & Zimogênio \\
\hline Fator Stuart-Prower & Fator X & 58.500 & 10,0 & Zimogênio \\
\hline $\begin{array}{l}\text { Antecedente de tromboplastina } \\
\text { plasmática }\end{array}$ & Fator XI & 160.000 & 4,8 & Zimogênio \\
\hline Fator de Hageman & Fator XII & 85.000 & 40,0 & Zimogênio \\
\hline Fator estabilizador de Fibrina & Fator XIII & 320.000 & 20,0 & Zimogênio \\
\hline Fator de Fletcher & Precalicreína & 90.000 & 40,0 & Zimogênio \\
\hline Fator de Fitzgerald* & $\begin{array}{l}\text { Cininogênio de } \\
\text { alto peso } \\
\text { molecular }\end{array}$ & 120.000 & 70,0 & Zimogênio \\
\hline
\end{tabular}

*Também conhecido como Williams, Flaujeac ou Fatores de Reid. NA- Não aplicável (proteína membrânica).

\section{3- PROCESSO HEMOSTÁTICO HEMOSTASIA PRIMÁRIA}

A hemostasia primária consiste na primeira fase da formação do trombo e seus quatro principais constituintes são o endotélio, as plaquetas, as micropartículas e o $\mathrm{FvW}^{5,61}$. Didaticamente é dividida em 4 fases: vasoconstricção, adesão, ativação e agregação plaquetária.

\section{1 - Vasoconstricção}

É o evento inicial transitório, que visa retardar a perda sanguínea extravascular e diminuir o fluxo sanguíneo local. A lesão vascular leva a eliminação da barreira física e metabólica decorrente do endotélio, com isso as substâncias vasoconstritoras (endotelina 1, serotonina e TXA2) podem agir diretamente nas células do músculo liso vascular ${ }^{12,3}$. Em relação aos vasos de pequeno calibre ocorre simplesmente um colabamento das paredes nos vasos ${ }^{31,63}$.

\section{2 - Adesão e ativação plaquetária}

Inicialmente a adesão da plaqueta ao vaso lesado ocorre pela interação do único receptor de plaqueta (GPIb $\alpha$ ), que não requer ativação prévia, ao $\mathrm{FvW}$ presente no subendotélio. Logo, via sinalização intracelular, 
as plaquetas são ativadas e outros receptores, entre eles as glicoproteínas (GPIb-II $\alpha$-V-IX) e integrinas $\left(\alpha_{\mathrm{II}} \beta_{3}\right.$ e $\left.\alpha_{2} \beta_{1}\right)$, ficam expostos para se ligarem ao FvW, ao colágeno e a fibronectina fato que intensifica a adesão plaquetária ao endotélio ${ }^{53,73}$.

O colágeno, o $\mathrm{FvW} \quad \mathrm{e}$ as micropartículas são os principais indutores da ativação plaquetária. Neste processo, o formato discoide das plaquetas é modificado para uma estrutura irregular, devido ao aumento na concentração de cálcio intracelular, que ativa o sistema contrátil actina-miosina $^{24}$. O aumento de cálcio ativa ainda a fosfolipase A2, que libera o ácido araquidônico da membrana plaquetária e dá início à síntese do TXA2, importante indutor da ativação e agregação plaquetária ${ }^{77}$. As plaquetas também são ativadas por meio da interação de trombina com a GPIb plaquetária $^{21}$.

Quando ativadas, as plaquetas liberam diversos constituintes dos grânulos eletrodensos e $\alpha$, entre eles estão os potentes vasoconstritores [serotonina, TXA2 e adenosina difosfato (ADP)], e os ativadores de plaquetas adicionais (trombina- $\alpha$, ativador plaquetário 4 e TXA2) ${ }^{10,28}$. Por fim, há liberação das prostaciclinas que levam à formação de uma fina monocamada de plaquetas na região da lesão vascular ${ }^{59,42,1,2}$.

\section{3 - Agregação plaquetária}

Durante a agregação plaquetária ocorre a formação do tampão hemostático primário no local da lesão vascular. Nessa fase, o principal evento é a alteração funcional das glicoproteínas e integrinas presentes na superfície das plaquetas que auxiliam na estabilização da agregação plaquetária ${ }^{36}$. Neste contexto, ocorre uma segunda ligação entre o FvW e o colágeno às integrinas plaquetárias $\left(\alpha_{\mathrm{IIb}} \beta_{3}\right.$ e $\left.\alpha_{2} \beta_{1}\right)$. A seguir, os receptores plaquetários GPIIb-III $\alpha$ e integrina $\alpha_{\mathrm{IIb}} \beta_{3}$ se ligam ao fibrinogênio solúvel, tornando a agregação plaquetária irreversível 46,4 .

O tampão hemostático primário é um evento transitório, que inibe momentaneamente o sangramento e fornece uma superfície pró-coagulante para os passos seguintes da cascata da coagulação. Entretanto, quando a lesão vascular é de pequena dimensão, este tampão é suficiente para coibir a hemorragia ${ }^{1,65,43}$.

\section{4- HEMOSTASIA SECUNDÁRIA OU COAGULAÇÃO SANGUÍNEA}

Durante a hemostasia secundária ocorre a ativação de uma série de reações enzimáticas envolvendo as proteínas prócoagulantes presentes no plasma que levam à formação do coágulo sanguíneo ${ }^{55}$.

\section{1 - Cascata clássica da coagulação sanguínea}

Em 1964, dois grupos independentes propuseram o modelo clássico da coagulação sanguínea $^{17,39}$. Esse modelo descreve cada 
fator da coagulação como proenzima que pode ser convertido em enzima ativada por meio de reações proteolíticas sequenciais. $\mathrm{O}$ modelo da "cascata da coagulação" dividiu as reações enzimáticas em dois sistemas distintos, a via intrínseca e a via extrínseca (Figura 2).

$\mathrm{Na}$ via intrínseca todos os componentes estão presentes na circulação sanguínea e se inicia após a interação do FXII com a superfície negativa do endotélio lesado $^{57,20,18}$. A molécula do CAPM atua como cofator do FXIIa, que converte a próenzima PC em calicreína. Logo, a calicreína acelera a conversão do FXII em FXIIa, sendo este capaz de se autoativar (feedback positivo). O FXIIa converte o FXI inativo à sua forma ativa. O FXIa se comporta como uma serino-protease e ativa o substrato FIX. O fator IXa juntamente com o FVIIIa (ativado pela trombina), fosfolipídios carregados negativamente (principais constituintes da membrana celular) e íons cálcio (sintetizados por plaquetas ativadas), forma o complexo tenase intríseco e cliva o fator $\mathrm{X}$ tornando-o $\operatorname{ativo~}^{64,9}$.

A via do sistema extrínseco é desencadeada por um fator externo ao sangue circulante, o FT (proteína de membrana celular do subendotélio) que juntamente com íons cálcio e FVIIa (ativado pelo FT) forma o complexo tenase extrínseco convertendo o FX na forma ativa ${ }^{51}, 70,40$. Ambas as vias convergem na "via comum" - ponto de ativação do fator $\mathrm{X}$. Logo, o complexo protrombinase, $\mathrm{FXa} / \mathrm{FVa} / \mathrm{Ca}^{2+} /$ fosfolipídios é responsável pela conversão da protrombina em trombina. A principal ação da trombina é catalisar a proteólise do fibrinogênio em monômeros de fibrina solúveis. A seguir, os monômeros de fibrina sofrem ação do complexo trombina/FXIIIa/Ca $\mathrm{Ca}^{2+}$ gerando, por fim, o coágulo de fibrina insolúvel ${ }^{33,19}$.

Apesar da importância desse conceito para fins diagnósticos, observações clínicas e experimentais demonstraram que a hipótese da "cascata da coagulação" não refletia completamente o evento da hemostasia in vivo 55 .

Por exemplo, a deficiência dos fatores VIII e IX não podia ser compensada pela ativação do FIX da via extrínseca ${ }^{35,48,7}$. Além disso, doenças genéticas que resultam na deficiência do FXII, precalicreína ou cininogênio de alto peso molecular não resultam em hemorragia, embora o tempo de tromboplastina parcial ativa seja prolongado. Esses e outros questionamentos conduziram a elaboração de um novo modelo que esclarece melhor a hemostasia in vivo $\mathrm{e}$ inclui a superfície das células e a interdependência das duas vias no processo de coagulação sanguínea $^{57}$.

\section{2 - Modelo atual da coagulação sanguínea}

O modelo atual da coagulação sanguínea, mostrado na Figura 3, sugere o envolvimento de três fases distintas: iniciação, amplificação e propagação que 
ocorrem na superfície da célula portadora do

FT e da plaqueta ativada ${ }^{32}$.

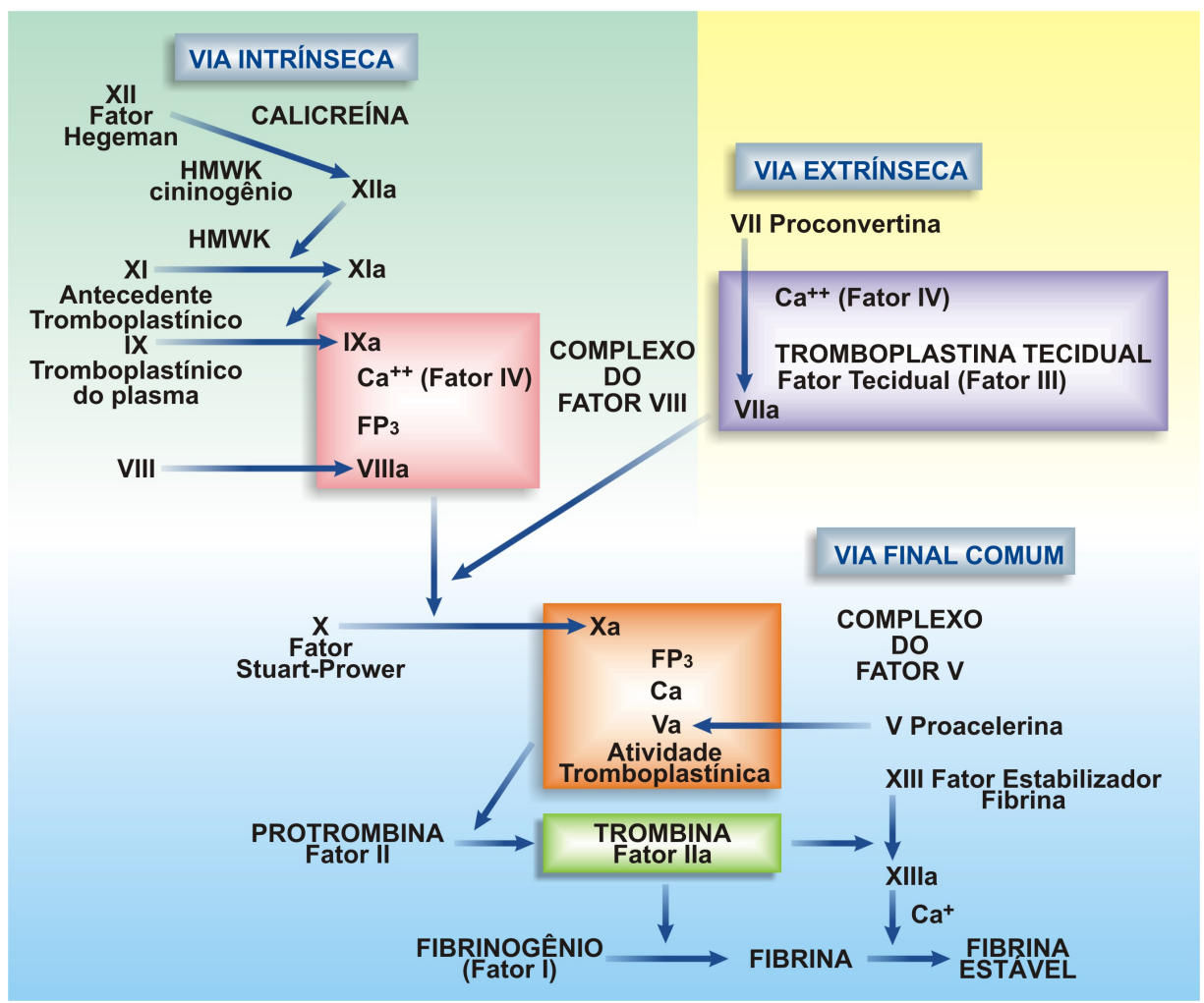

Figura 2 - Modelo Clássico da Cascata da Coagulação Sanguínea. Este modelo divide a cascata da coagulação em duas vias: via intrínseca e via extrínseca. A via intrínseca é desencadeada pelo contato com plaquetas ativadas ou componentes do tecido endotelial. Essa interação entre os fatores XII, calicreína e o cininogênio de alto peso molecular resulta na ativação do fator XII que converte o fator XI de sua forma zimogênica para sua forma enzimática (FXIa). A seguir, o fator XIa ativa o fator IX e o FIXa juntamente com $\mathrm{Ca}^{2+}$, FP3 e o FVIIIla formam um complexo que ativa o fator X. Na via extrínseca, o fator tecidual inicia a cadeia de eventos ao formar um complexo com o FVIIa. Este complexo ativa o fator X que se encontra na intersecção das duas vias levando a formação do complexo FXa/FVa. Com isso ocorre a ativação de protrombina que age convertendo o fibrinogênio em fibrina (Figura modificada de Roberts et al, $2004^{58}$.

\subsection{1 - Fase de iniciação}

Ocorre nas células que expressam o FT em sua superfície: célula endotelial, monócito, fibroblasto, entre outras. Mediante a exposição do FT na circulação pela lesão ou ativação das células endoteliais, monócitos, ou micropartículas oriundas de vários tipos de células forma-se um complexo entre FT e pequenas quantidades de FVIIa $^{51,47}$. Esse complexo formado TF/FVIIa inicia a coagulação por ativar o fator $\mathrm{X}$ e fator IX. O fator Xa ativa o fator V. Após a ativação o fator $\mathrm{Va}$ associa-se ao fator $\mathrm{Xa}$ e forma $\mathrm{o}$ complexo protrombinase $\mathrm{FXa} / \mathrm{FVa}$. Esse complexo é capaz de converter a protrombina (II) em traços de trombina (IIa $)^{60}$. Além disso, o fator IXa gerado pode se deslocar para outra célula ou para a superfície das plaquetas tornando-as ativas e assim iniciar a fase de amplificação da coagulação. 


\subsection{2 - Fase da amplificação}

Ocorre na superfície das plaquetas. $\mathrm{O}$ traço de trombina gerado na fase de iniciação desempenha 4 reações principais: 1) ativa as plaquetas; 2) ativa o fator $\mathrm{V}$ na superfície das plaquetas formando o fator $\mathrm{Va} ; 3$ ) dissocia o complexo FVIII:FvW e 4) ativa o FXI na superfície das plaquetas formando o FIXa . O FvW livre participa da adesão e agregação plaquetária no local da lesão vascular. Estando os fatores ativados na superfície das plaquetas inicia-se a fase de propagação.

\subsection{3 - Fase de propagação}

Também ocorre na superfície das plaquetas. O IXa ativado na fase de iniciação associa-se ao VIIIa liberado na fase de amplificação e forma o complexo tenase FIXa/FVIIIa. O complexo tenase, por sua vez, ativa o fator X. Esse fator ativado Xa associase ao seu cofator $\mathrm{Va}$ e forma o complexo protrombinase FXa/FVa. Nessa fase, grandes quantidades de plaquetas são recrutadas para o local da lesão e são formados inúmeros complexos tenase (FIXa/FVIIIa) e protrombinase $(\mathrm{FXa} / \mathrm{FVa})$ na superfície das mesmas. Consequentemente, grandes quantidades de protrombina são convertidas em trombina que resulta na clivagem de fibrinogênio em monômeros de fibrina. A fibrina se polimeriza e resulta na formação de coágulo estável de fibrina ${ }^{33,58,8}$.

\section{5- FIBRINÓLISE OU HEMOSTASIA TERCIÁRIA}

Após a formação do trombo, o mesmo deve ser removido para o restabelecimento do lúmen vascular e do fluxo sanguíneo. Nesse sentido, a fibrinólise é o processo que realiza gradualmente a remoção dos depósitos de fibrina. Ela é regulada por proteínas ativadoras serino-proteases, metaloproteinases (MMPs) e proteínas inibidoras como a superfamília de serpinas ${ }^{13,66}$.

O primeiro passo da fibrinólise é a ativação do plasminogênio (PLG), um precursor circulante inativo, que é convertido em plasmina por duas vias de ativação. A primeira via de ativação é mediada pelo ativador tissular de PLG - tPA (do inglês, tissue-type plasminogen activator) que é produzido pela célula endotelial, principalmente após a ativação da coagulação. O tPA converte o PLG em plasmina. Esta última é uma serino protease que degrada a rede de fibrina em produtos de degradação solúveis (PDF). A afinidade entre tPA e PGL é baixa na ausência de fibrina, contudo quando há formação de coágulo de fibrina essa afinidade se eleva, potencializando a ação do tPA. Adicionalmente, a anexina 2, secretada pelas células endoteliais, se liga tanto ao PLG quanto ao tPA, agindo como cofator na geração de plasmina ${ }^{67}$. Além disso, cerca de $15 \%$ da plasmina circulante é gerada via proteínas pró-coagulante: Calicreína e FXIa, que ativam diretamente o $\mathrm{PLG}^{16}$. 


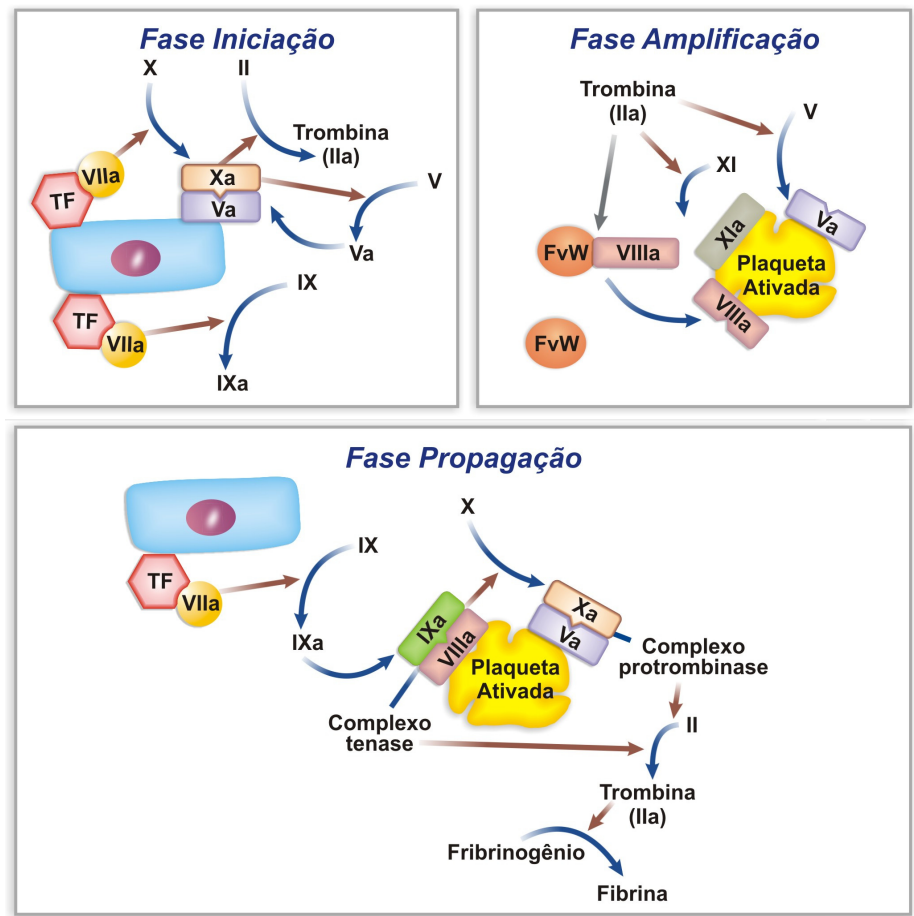

Figura 3 - Novo Modelo da Cascata de Coagulação Sanguínea. De acordo com esse modelo, o processo hemostático ocorre sobre superfícies celulares distintas em uma série de três etapas simultâneas. A primeira etapa é a fase de iniciação: células endoteliais, micropartículas ou monócitos expõem o FT que se associa ao FVIIa formando o complexo FT-VIIa. A seguir, o complexo TF-VIla ativa o fator X formando fator Xa. O fator Xa ativa o fator V formando o fator Va. Esse associa-se ao fator Xa e converte a protrombina (fator II) em trombina. Além disso, na fase o complexo FT-VIIa ativa o fator IX formando fator IXa. Na fase de amplificação, o traço de trombina gerado ativa a plaqueta. Na superfície da plaqueta ativada são ativados 3 fatores pela ação do traço de trombina gerado na fase de iniciação: fatores Va, XIa e VIIIa. Esse último é ativação após dissociar-se do complexo FvW/FVIIIa. Com esses fatores ativados na superfície da plaqueta ativada inicia-se a fase de propagação. Nessa fase são formadas grandes quantidades de dois complexos: tenase (IXa/VIIIa) e protrombinase (Xa/Va). Esses complexos ativam a protrombina formando trombina. Esta última converte fibrinogênio em fibrina, a qual após a polimerização forma o coágulo de fibrina. (Modificado de Hoffman, 2004).

A segunda via de ativação ocorre pela ligação do uPA (do inglês, urokinase-type plasminogen activator- ativador de plasminogênio tipo uroquinase) que também é produzido pela célula endotelial após a ativação da coagulação. O uPA converte o PLG em plasmina, e esta ativa as MMPs. Essas metaloproteinases estão envolvidas na degradação da matriz extracelular e no remodelamento tecidual.

De forma equilibrada, a dissolução da fibrina também é regulada por proteínas inibidoras, que atuam diretamente nos ativadores de PLG (do inglês plasminogen activator inhibitors $\quad-$ inibidor de plasminogênio 1 [PAI -1] e inibidor de plasminogênio 2 [PAI-2]) e pela $\mathrm{TM}^{78,13}$. Existem ainda proteases inibidoras que atuam na plasmina, como a $\alpha 2$-antiplasmina e $\alpha 2$ macroglobulina. $\mathrm{O}$ sítio de ligação utilizado para $\alpha 2$-antiplasmina e plasmina, é o mesmo sítio utilizado para fibrina e plasmina. Logo, para que a $\alpha 2$-antiplasmina iniba a plasmina, é necessário que inibidores da fibrinólise 
ativados por trombina (do inglês, thrombin activatable fibrinolysis inhibitor - TAFI), removam os resíduos de lisina do carbono terminal da fibrina, liberando dessa forma o sitio de ligação para plasmina- $\alpha 2-$ $\operatorname{antiplasmina~}^{56,37,41}$.

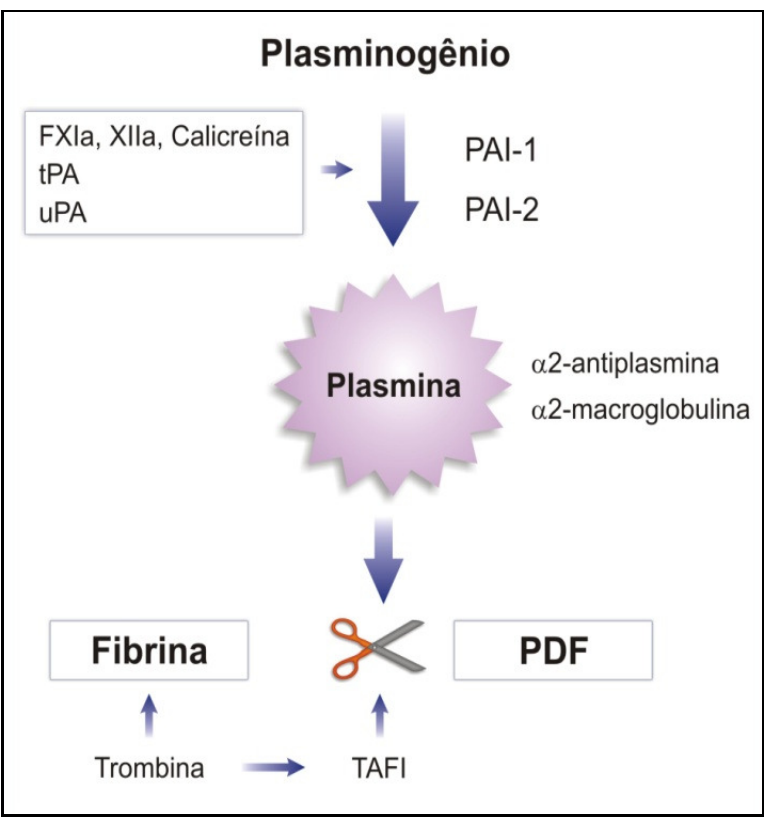

Figura 4 - Sistema Fibrinolítico. A conversão do plasminogênio em plasmina é ativada pelos constituintes da fibrinólise: tPA e uPA. Consequentemente há degradação da rede de fibrina e geração dos PDF (produtos de degradação de fibrina). Este processo pode ser modulado pelos inibidores dos ativadores do plasminogênio 1 e 2 (PAI-1 e PAI-2), neutralizadores de plasmina $\alpha 2$-antiplasmina, $\alpha 2$ macroglobulina e sistema TAFI.

\section{6- CONCLUSÃO}

Neste trabalho procuramos fornecer uma visão concisa dos conceitos atuais sobre coagulação e hemostasia. Primeiramente abordamos o conceito clássico da coagulação que sugere a ativação sequencial de diversas pró-enzimas. Em seguida, abordamos o conceito atual que propõe o mecanismo de coagulação dependente das superfícies celulares e não envolve todos os fatores previamente propostos, como por exemplo, o fator XII. Abordamos ainda a contribuição da superfície endotelial, micropartículas e plaquetas no processo de coagulação. Todo este processo ocorre através de passos sequenciais, sendo referido como hemostasia primária e secundária. Após o reparo da lesão vascular, o sistema fibrinolítico ou hemostasia terciária atua no restabelecimento do fluxo sanguíneo, impedindo a coagulação excessiva e desnecessária.

A perda do equilíbrio dinâmico destas reações tem como consequência o aparecimento de distúrbios hemorrágicos graves que podem comprometer a vida do paciente. A coagulação insuficiente pode se manifestar em desordens tais como a hemofilia $\mathrm{A}$, hemofilia $\mathrm{B}$ e a doença de von Willebrand. Por outro lado, um aumento excessivo na coagulação pode causar complicações como, por exemplo, o aparecimento de trombose.

Espera-se que o conhecimento do novo modelo da cascata da coagulação, associado ao conhecimento mais complexo do papel da célula endotelial, micropartículas e plaquetas no mecanismo de coagulação, bem como, os avanços no conhecimento do genoma humano, traga avanços significativos na compreensão dos mecanismos de hemostasia. Espera-se ainda que tais conquistas permitam o desenvolvimento de novas metodologias de diagnóstico e 
intervenções terapêuticas mais precisas no tratamento de hemofilia A e B.

\section{AGRADECIMENTOS}

Fundação de Amparo à Pesquisa do Estado de São Paulo (FAPESP - processo $n^{\circ}$ 1998/14247-6), Financiadora de Estudos e Projetos (FINEP - processo $n^{\circ}$ 01.07.0652.00)

e Conselho Nacional de Desenvolvimento Científico e Tecnológico $\left(\mathrm{CNPq}-\right.$ processo $\mathrm{n}^{\circ}$ $314458 / 2009-3$ e 2008/57877-3). Os autores agradecem à Fernanda Teresinha Udinal pela revisão ortográfica e à Sandra Navarro Bresciani pelas ilustrações deste trabalho.

\section{REFERÊNCIAS}

1 Andrews RK, Berndt MC. Platelet physiology and thrombosis. Thromb Res. 2004; 114:447-53.

2 Andrews RK, Berndt MC. Platelet adhesion: a game of catch and release. $\mathrm{J}$ Clin Invest. 2008; 118(9):3009-11.

3 Becker BF, Heindl B, Kupatt C, Zahler S. Endothelial function and hemostasis. Z Kardiol. 2000; 89:160-7.

4 Bennett JS, Berger BW, Billings PC. The structure and function of platelet integrins. J Thromb Haemost. 2009 Jul;7 Suppl 1:200-5.

5 Berckmans RJ, Neiuwland R, Böing AN, Romijn FP, Hack CE, Sturk A. Cellderived microparticles circulate in healthy humans and support low grade thrombin generation. Thromb Haemost. 2001 Apr;85(4):639-46.

6 Britten MB, Zeiher AM, Schächinger V. Clinical importance of coronary endothelial vasodilator dysfunction and therapeutic options. J Intern Med. 1999; 245:315-27.

7 Butenas S, Mann KG. Blood coagulation. Biochemistry (Mosc). 2002; 67:3-12.

8 Butenas S, Orfeo T, Gissel MT, Brumel KE, Mann KG. The significance of circulating factor IXa in blood. J Biol Chem. 2004; 279: 22875-22882.

9 Camire RM, Larson PJ, Stafford DW, Katherine A. High. Enhanced $\gamma$ Carboxylation of Recombinant Factor X Using a Chimeric Construct Containing the Prothrombin Propeptide.

Biochemistry. 2000; 39:14322-9.

10 Cerletti C, De GG. Current concepts of platelet physiopathology.

Int.J.Artif.Organs, 1993; 16:205-208.

11 Chironi GN, Boulanger CM, Simon A, Dignat-George F, Freyssinet JM, Tedgui A. Endothelial microparticles in diseases. Cell Tissue Res. 2009 Jan;335(1):143-51. Epub 2008 Nov 7. Review.

12 Cines DB, Pollak ES, Buck CA, Loscalzo J, Zimmerma GA, McEver RP, Pober JS, Wick TM, Konkle BA, Schwartz BS, Barnathan ES, McCrae KR, Hug BA, Schimidt AM, Stern DM. Endothelial cells in physiology and in the pathophysiology of vascular disorders. Blood, 1998; 91:3527-3561.

13 Collen D. The plasminogen (fibrinolytic) system. Thromb Haemost. 1999; 82:259. 70.

14 Colman RW; Hirsh J; Marder VJ; Clowes AW. Overview of coagulation, fibrinolysis, and their regulation. In: COLMAN RW; HIRSH J; MARDER VJ; CLOWES AW \& GEORGE JN, eds. Hemostasis and thrombosis. Basic principles and clinical practice, 4th ed, Lippincott; Williams \& Wilkins, Philadelphia, p. 17-20, 2001.

15 Coombe DR, Kett WC. Heparan sulfateprotein interactions: therapeutic potential 
through structure-function insights. Cell Mol Life Sci. 2005 Feb;62(4):410-24.

16 Cesarman-Maus G, Hajjar KA. Molecular mechanisms of fibrinolysis. Br J Haematol. 2005 May;129(3):307-21.

17 Davie EW, Ratnoff OD, Waterfall sequence for intrinsic blood clotting. Science. 1964; 145:1310-1312.

18 Davie EW, Fujikawa K, Kisiel W. The coagulation cascade: initiation, maintenance, and regulation. Biochemistry. 1991; 30:10363-10370.

19 Davie EW. Biochemical and molecular aspects of the coagulation cascade. Thromb.Haemost. 1995; 74:1-6.

20 Davie EW. A brief Historical review of the waterfall/cascade of blood coagulation. J Biolog Chemist. 2003; 278: 50819-50832.

21 De Marco L, Mazzucato M, Masotti A, Ruggeri ZM. Localization and characterization of an alpha-thrombinbinding site on platelet glycoprotein $\mathrm{Ib}$ alpha. J Biol Chem. 1994 Mar 4;269(9):6478-84.

22 Esmon CT. Does Inflammation Contribute to Thrombotic Events?

Haemostasis. 2000; 30:34-40.

23 Franco, R.F. Fisiologia da Coagulação, anticoagulação e fibrinólise. Medicina, Ribeirão Preto. 2001; 34:229-237.

24 Gaetano G. Historical overview of the role of platelets in hemostasis and thrombosis. Haematologica. 2001; 86:349-56.

25 George FD. Microparticles in vascular diseases. Thromb Res. 2008;122 ,Suppl 1:S55-9.

26 Gilbert GE, Sims PJ, Wiedmer T, Furie B, Furie BC, Shattil SJ. Platelet-derived microparticles express high affinity receptors for factor VIII. J Biol Chem 1991; 266: 17261-8.
27 Goto S. Blood constitution: platelet aggregation, bleeding, and involvement of leukocytes. Rev Neurol Dis. 2008; 5:S227.

28 Gralnick HR, Williams S, McKeown LP, Hansmann K, Fenton JW 2nd, Krutzsch H. High-affinity alpha-thrombin binding to platelet glycoprotein Ib alpha: identification of two binding domains. Proc Natl Acad Sci USA. 1994 Jul 5;91(14):6334-8.

29 Higashi S, Iwanaga S. Molecular interaction between factor VII and tissue factor. Int J Hematol. 1998; 67:229-41.

30 Hinsbergh VW. The endothelium: vascular control of haemostasis. Eur $\mathbf{J}$ Obstet Gynecol Reprod Biol. 2001; 95:198-201.

31 Hirschi KK, D'Amore PA. Pericytes in the microvasculature. Cardiovasc Res. 1996; 32:687-98.

32 Hoffman M, Monroe D. A cell-based model of hemostasis. Trombosis and Haemostasis. 2001; 85:958-965.

33 Hoffman M. Remodeling the blood coagulation cascade.

J.Thromb.Thrombolysis. 2003; 16:17-20.

34 Hugel B, Martínez MC, Kunzelmann C, Freyssinet JM. Membrane microparticles: two sides of the coin. Physiology (Bethesda). 2005; 20:22-27.

35 Kirchhofer D, Nemerson Y. Initiation of blood coagulation: the tissue factor/factor VIIa complex. Curr.Opin.Biotechnol. 1996; 7: 386-391.

36 Kulkarni S, Dopheide SM, Yap CL, Ravanat C, Freund M, Mangin P, Heel KA, Street A, Harper IS, Lanza F, Jackson SP. A revised model of platelet aggregation under flow. Cell. 2000; 94:657-666.

37 Redlitz A, Plow EF. Receptors for plasminogen and t-PA: an update. 
Baillieres Clin Haematol. 1995

Jun;8(2):313-27.

38 Löwenberg E.C., Meijers J.C., Levi M., (2010) Platelet-vessel wall interaction in health and disease. Neth J Med. Jun;68(6):242-51

39 MacFarlane RG. An enzyme cascade in the blood clotting mechanism and its function as a biochemical amplifier. Nature. 1964; 202: 498-499.

40 Manly DA, Boles J, Mackman N.,(2010) Role of Tissue Factor in Venous Thrombosis. Annu Rev Physiol. 2010 Aug 3

41 Marx PF. Thrombin-activatable fibrinolysis inhibitor. Curr Med Chem. 2004; 11(17):2335-48. Review.

42 Maxwell MJ, Dopheide SM, Turner SJ, Jackson SP. Shear induces a unique series of morphological changes in translocating platelets: effects of morphology on translocation dynamics. Arterioscler Thromb Vasc Biol. 2006; 26:663-9.

43 Maxwell MJ, Westein E, Nesbitt WS, Giuliano S, Dopheide SM, Jackson SP. Identification of a 2-stage platelet aggregation process mediating sheardependent thrombus formation. Blood. 2007; 15:566-76.

$44 \mathrm{McNicol}$ A, Israels SJ. Platelet dense granules: structure, function and implications for haemostasis. Thromb.Res. 1999; 95:1-18.

45 Morelli VM. Estrutura e função das plaquetas e das células endoteliais. In: Zago, A.M.; Falcão, R.P.; Pasquini, R. Hematologia Fundamentos e Prática. Editora Atheneu, 2001. p. 731-737.

46 Moroi M, Jung SM, Nomura S, Sekiguchi S, Ordinas A, Diaz-Ricart M. Analysis of the involvement of the von Willebrand factor-glycoprotein $\mathrm{Ib}$ interaction in platelet adhesion to a collagen-coated surface under flow conditions. Blood. 1997;90:4413-4424.

47 Morrissey JH, Macik BG,

Neuenschwander PF, et al. Quantitation of activated factor VII levels in plasma using a tissue factor mutant selectively deficient in promoting factor VII activation. Blood 1993; 81: 734-44.

48 Nemerson Y. The tissue factor pathway of blood coagulation. Semin.Hematol. 1992; 29:170-176.

49 Pearson JD. Endothelial cell biology. In: Bloom, A.L.; Forbes, C.D.; Thomas, D.P.; Tuddennham, E.G.D. Haemostasis and thrombosis. Churchill Livingstone, Third Edition, 1994. p. 219-232.

50 Pearson JD. Endothelial cell function and thrombosis. Baillieres Best Pract Res Clin Haematol. 1999; 12:329-341.

51 Price GC, Thmpson SA, Kam PC. Tissue factor and tissue factor pathway inhibitor. Anaesthesia. 2004; 59:483-492.

52 Rapaport SI, Rao LV. The tissue factor pathway: how it has become a "prima ballerina". Thromb Haemostr 1995; 74:717.

53 Reininger AJ. Function of von Willebrand factor in haemostasis and thrombosis. Haemophilia. 2008 Nov;14 Suppl 5:1126.

54 Reitsma S, Slaaf DW, Vink H, van Zandvoort MA, oude Egbrink MG. The endothelial glycocalyx: composition, functions, and visualization. Pflugers Arch. 2007; 454:345-59.

55 Riddel JP, Aouizerat BE, Miaskowski C, Lillicrap DP. Theories of Blood Coagulation. Journal of Pediatric Oncology Nursing. 2007; 24:123-131.

56 Rijken DC, Sakharov DV. Basic principles in thrombolysis: regulatory role of plasminogen. Thromb Res. 2001; 30:103 Suppl 1:S41-9. Review. 
57 Roberts HR, Monroe DM, Oliver JA, Chang JY, Hoffman M. Newer concepts of blood coagulation. Haemophilia. 1998; 4:331-334.

58 Roberts HR, Monroe DM, Escobar MA. Current concepts of hemostasis: implications for therapy. Anesthesiology. 2004; 100:722-730.

59 Ruggeri ZM. Platelets in atherothrombosis. Nat.Med. 2002; 8:1227-1234.

60 Sims PJ, Faioni EM, Wiedmer T, Shattil SJ. Complement proteins C5b-9 cause release of membrane vesicles from the platelet surface that are enriched in the membrane receptor for coagulation factor $\mathrm{Va}$ and express prothrombinase activity. $\mathrm{J}$ Biol Chem 1988; 263: 18205-12.

61 Spronk HM, Govers-Riemslag JW, ten Cate $\mathrm{H}$. The blood coagulation system as a molecular machine. Bioessays. 2003; 25:1220-1228.

62 Stenberg PE, Levin J. Ultrastructural analysis of acute immune thrombocytopenia in mice: dissociation between alterations in megakaryocytes and platelets. J Cell Physiol. 1989; 141:160-169.

63 Stenberg PE, Hill RJ. Platelets and megakaryocytes. In: Lee GR, Foerster J, Greer J, Lukens J, Paraskevas F, Rodgers GM, eds. Wintrobe's Clinical Hematology, 10th edition. Philadelphia, PA: Williams \& Wilkins, 1998;615-660.

64 Straight DL, Sherrill GB, Noyes CM, Trapp HG, Wright SF, Roberts HR, Hiskey RG, Griffith MJ. Structural and functional characteristics of activated human factor IX after chemical modification of gamma-carboxyglutamic acid residues. J Biol Chem. 1985; 260:2890-3.

65 Suhasini K, Sacha MD, Yap CL, Ravanat C,Freund M,Mangin P, Heel CA, Street A, Harper IS, Lanza F, Jackson SP. A revised model of platelet aggregation under flow. Cell.2000; 94:657-666.

66 Takada A, Takada Y, Urano T. The physiological aspects of fibrinolysis. Thromb Res. 1994; 76(1):1-31.

67 Hajjar KA, Jacovina AT, Chacko J. An endothelial cell receptor for plasminogen/tissue plasminogen activator. I. Identity with annexin II. J Biol Chem. 1994 Aug 19;269(33):21191-7.

68 Troy GC. An overview of hemostasis. Vet Clin North Am Small Anim Pract. 1988; 8:5-20.

69 Tuddennham EGD, Laffan MA. Inherited bleeding disorders. In: Hoffbrand, A.V.; Lewis, S.M.; Tuddennham, E.G.D. Postgraduate Haematology. Fourth Edition, 1999, p. 612-635.

70 Walsh PN, Ahmad SS. Proteases in blood clotting. Essays Biochem 2002; 38:95111.

71 Weiskopf RB. The use of recombinant activated coagulation factor VII for spine surgery. Eur.Spine J. 2004; 13: S83-S88.

72 White JG, Krumwiede M, CockingJohnson D, Escolar G. Influence of combined thrombin stimulation, surface activation, and receptor occupancy on organization of GPIb/IX receptors on human platelets. Br J Haematol. 1994; 88:137-148.

73 Wohner N. Role of cellular elements in thrombus formation and dissolution. Cardiovasc Hematol Agents Med Chem. 2008; 63:224-8.

74 Wright IS. The nomenclature of blood clotting factors. Can Med Assoc J. 1962 Feb 24;86:373-4.

75 Ronald A. Hutton, Michael A. Laffan and Edward G.D. Tuddenham. Normal Haemostasis.Postgraduate Haematlology. Fouth Edition, 550-80, 1999. Butterworth Heinemann. A. Victor Hoffbrand, 
S.Michell Lewis, Edward G.D.

Tuddenham.

76 Baj-Krzyworzeka M, Majka M, Pratico D, Ratajczak J, Vilaire G, Kijowski J, Reca

R, Janowska-Wieczorek A, Ratajczak

MZ. Platelet-derived microparticles

stimulate proliferation, survival, adhesion, and chemotaxis of hematopoietic cells.

Exp Hematol. 2002 May;30(5):450-9.

77 Barry OP, Pratico D, Lawson JA,

FitzGerald GA. Transcellular activation of platelets and endothelial cells by bioactive lipids in platelet microparticles. J Clin

Invest. 1997 May 1;99(9):2118-27.

$78 \mathrm{Wu}$ KK, Thiagarajan P. Role of endothelium in thrombosis and hemostasis. Annu. Rev. Med. 1996; 47:315-31. 